\title{
Interstitial Ectopic Pregnancy and Laparoscopic Removal of the Interstitial Portion of the Tube: the New Frontier?
}

\author{
Ping Ling $\mathrm{Ho}^{\mathrm{a}, \mathrm{c}}$, Janice Tung ${ }^{\mathrm{a}}$, Siraj Shahul Hameed Mohamed ${ }^{\mathrm{b}}$, \\ Bernard Su Min Chern ${ }^{b}$
}

\begin{abstract}
We present a case of a 40-year-old lady with 9 weeks of amenorrhea diagnosed with an interstitial pregnancy and multiple uterine fibroids. She underwent laparoscopic salpingectomy including the interstitial part of fallopian tube with products of conception without myomectomy and cornuostomy. Intra-operative blood loss was minimal. She was followed up post-operatively with weekly serum beta human chorionic gonadotropin (serum $\beta$-hCG), which down-trended optimally at day 23 of surgery. Histopathology confirmed ectopic pregnancy with underlying salpingitis isthmica nodosa. Patient recovered well.
\end{abstract}

Keywords: Submyomatous interstitial pregnancy; Laparoscopic salpingectomy; Salpingitis isthmica nodosa

\section{Introduction}

Laparoscopic salpingectomy including interstitial fallopian tube without myomectomy and cornuostomy may be a new frontier in management of interstitial pregnancy, especially those with overlying large fibroid at the cornual region. In addition, this method reduces the thermal injury of myometrium. As a result, it may reduce the morbidity associated with the conventional wedge resection or cornuostomy, such as blood loss, and potential risks of uterine rupture in future pregnancy. Using this method, interstitial pregnancy with overlying uterine fibroid can also be managed successfully as in our case without the need of myomectomy. To the best of our knowledge, this is the first description of interstitial pregnancy with overlying uterine fibroid and successful removal of interstitial pregnancy via laparoscopic salpingectomy without cornuos-

Manuscript submitted May 28, 2019, accepted June 19, 2019

Published online July 21, 2020

aDepartment of Obstetrics and Gynaecology, KK Women's and Children's Hospital, Singapore

'Department of Minimally Invasive Surgery, KK Women's and Children's Hospital, Singapore

${ }^{c}$ Corresponding Author: Ping Ling Ho, 100 Bukit Timah Road, 229899 Singapore. Email: pingling.ho@gmail.com

doi: https://doi.org/10.14740/jmc3317 tomy and myomectomy.

\section{Case Report}

A 40-year-old lady, gravida 3 para 1, presented with 9 weeks of amenorrhea with history of intermittent right iliac fossa pain, associated with abdominal bloatedness and per vaginal spotting. She had one previous cesarean section, and no other medical or surgical history of note. She was clinically well and stable. Vitals were stable at presentation with blood pressure of 102/54 mm Hg and heart rate of $82 / \mathrm{min}$. Abdomen was normal, soft, non-tender. No signs of acute abdomen were noted. Vaginal examination revealed minimal amount of stale blood, cervical os otherwise was closed and tubular. Ultrasonographic imaging revealed a cystic mass measuring $3.1 \times$ $2.4 \times 2.3 \mathrm{~cm}$ at left cornu region, with surrounding peripheral vascularity, multiple uterine fibroids, and largest subserosal at anterior wall measuring $3.7 \mathrm{~cm}$. No pelvis fluid was noted. Serum human chorionic gonadotrophin (beta-hCG) at presentation was $31,750 \mathrm{IU} / \mathrm{mL}$. Couple was counseled regarding options of management for interstitial pregnancy. This was an unplanned pregnancy and she had completed family. She opted for bilateral salpingectomy as treatment for her current condition, also for permanent sterilization. Diagnostic laparoscopy was done and the intra-operative findings showed a $4 \mathrm{~cm}$ subserosal fibroid at the left cornu region, overlying the non-ruptured interstitial pregnancy (Fig. 1). Bilateral fallopian tubes and ovaries appeared normal. No evidence of FitzHugh-Curtis was noted. Laparoscopic salpingectomy including removal of interstitial fallopian tube was done without myomectomy and cornuostomy. We observed minimal blood loss and thermal injury to the myometrium. We were able to conserve more myometrium than the traditional method of cornuostomy techniques. Intra-operative blood loss was minimal, hemoglobin was $12.5 \mathrm{~g} / \mathrm{dL}$, compared to $13.1 \mathrm{~g} / \mathrm{dL}$ preoperatively. Beta-hCG was $15,583.9 \mathrm{IU} / \mathrm{mL}$ on post-operative day 2 (51\% reduction). Her histopathology revealed a bilateral isthmica salpingitis nodosa, product of conception in right fallopian tube. Patient recovered well, and was discharged stable on post-operative day 2 . Subsequent outpatient monitoring revealed optimal reduction of beta-hCG, at $41.8 \mathrm{IU} / \mathrm{mL}$ on post-operative day 17 and $15.5 \mathrm{IU} / \mathrm{mL}$ on post-operative day 23 . Her urine pregnancy test subsequently was negative. Patient remained well and stable. 


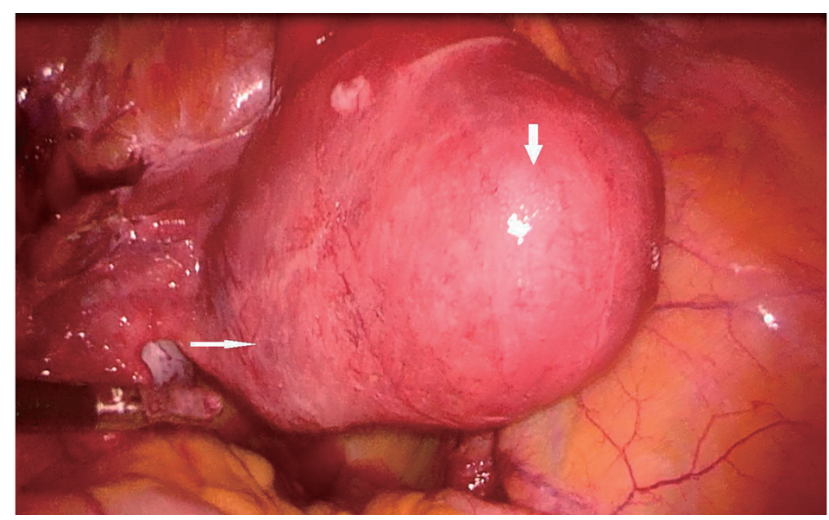

Figure 1. Interstitial pregnancy (white arrow) with overlying myoma (thick white arrow).

\section{Discussion}

Interstitial pregnancy is a rare but potentially life-threatening gynecological condition. Interstitial pregnancy accounts for $2-4 \%$ of ectopic pregnancies. Due to the location and distensibility of the myometrium, interstitial pregnancy is previously believed to present at a relatively later gestational age around 7 to 12 weeks gestation, compared to other types of ectopic pregnancies. Despite its rarity, it poses a diagnostic and therapeutic challenge, with higher mortality rate of $2-2.5 \%$, compared to other types of ectopic pregnancies. Late diagnosis leads to cornual rupture, significant hemorrhage, hypovolemia, shock, or even death.

The fallopian tubes arise from the uterine corpus posterior to bilateral round ligaments, and the lumen communicates with uterine cavity and intra-abdominal cavity. The fallopian tube can be divided into four distinct portions, namely interstitial, isthmus, ampulla and fimbriae. The interstitial portion is the most proximal part of the fallopian tube that lies within the muscular wall of the uterine corpus. It runs a slightly tortuous course, extends oblique superiorly and outward from the uterine corpus and measures approximately $1-2 \mathrm{~cm}$ long and $0.7 \mathrm{~mm}$ wide within the muscular uterine wall. It is lined by an outer longitudinal smooth muscle muscularis layer and an inner circular muscular fiber. The principal arterial blood supply of the fallopian tube arises mainly from uterine artery, which bifurcates and anastomoses with ovarian artery at the inferior of fallopian tube. Farrer-Brown et al showed that the uterine arteries run a tortuous course between the two layers of the broad ligament along the lateral side of the uterus and turn laterally at the junction of the uterus and fallopian tube, run toward the hilum of the ovary and terminate by joining the ovarian arteries (Figs. 2 and 3) [1]. Anastomoses between uterine and ovarian arteries in the mesosalpinx are variable but always present. Understanding this vascularity of anatomy is more important to reduce blood loss during the above surgical method of removing the interstitial portion of the fallopian tube.

Pregnancy that lies within interstitial fallopian tube is called interstitial pregnancy. As it receives abundant supply from both the uterine and ovarian vessels, rupture at this region often leads to severe hemorrhage, and death in worst case sce-

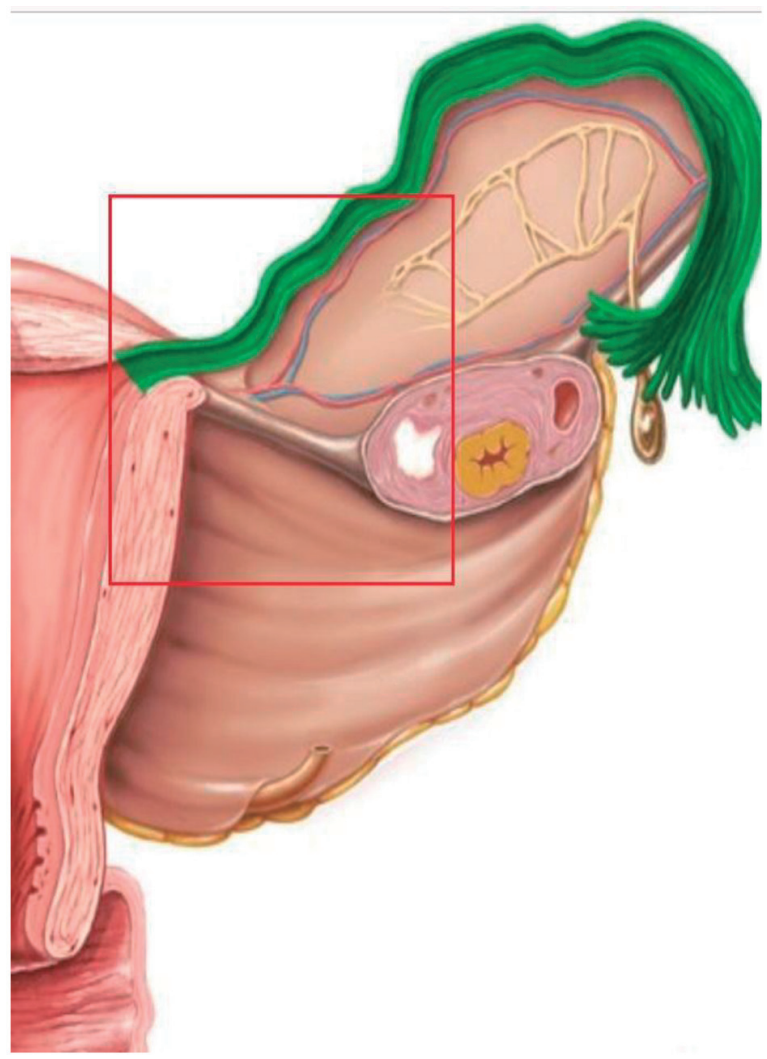

Figure 2. Vasculature of fallopian tube, anastomosis of uterine artery and ovarian artery (credit to www.kenhub.com).

nario. It carries a higher risk of maternal mortality compared to ectopic pregnancy at other sites of fallopian tubes.

Clinically, risk factors for interstitial pregnancy are similar to other types of ectopic pregnancy, except that previous history of ipsilateral salpingectomy which is a risk factor specific to interstitial pregnancy [2]. Major cause of ectopic pregnancy is underlying tubal anatomy disruption from infection, surgery, tumor, or congenital anomalies. Disrupted anatomical structure may be accompanied by damaged ciliary activity resulting in functional impairment. Previous tubal surgery and previous ectopic pregnancy remained the highest risk factor for ectopic pregnancy. It is observed that there is higher recurrence in interstitial pregnancy resulting from assisted conception, tubal pathology and those who underwent non-invasive management such as medical treatment. An association of recurrent ectopic pregnancy with uterine fibroids has also been described $[3,4]$.

Interstitial pregnancy is easily mistaken as intrauterine pregnancy due to its location in the muscular wall of the uterus. A common nomenclature often wrongly used is cornual pregnancy. Sonographic imaging thus plays an important role in making a correct diagnosis as the management and outcome differ. In an expert hand, the diagnosis can be established in $71 \%$ of the cases via transvaginal ultrasound [5]. A sensitivity and specificity of $80 \%$ and $99 \%$ has been reported [3]. Use of color Doppler of 3D and 4D ultrasound scanning may also improve the sensitivity of diagnosis [6,7]. Sonographic signs 


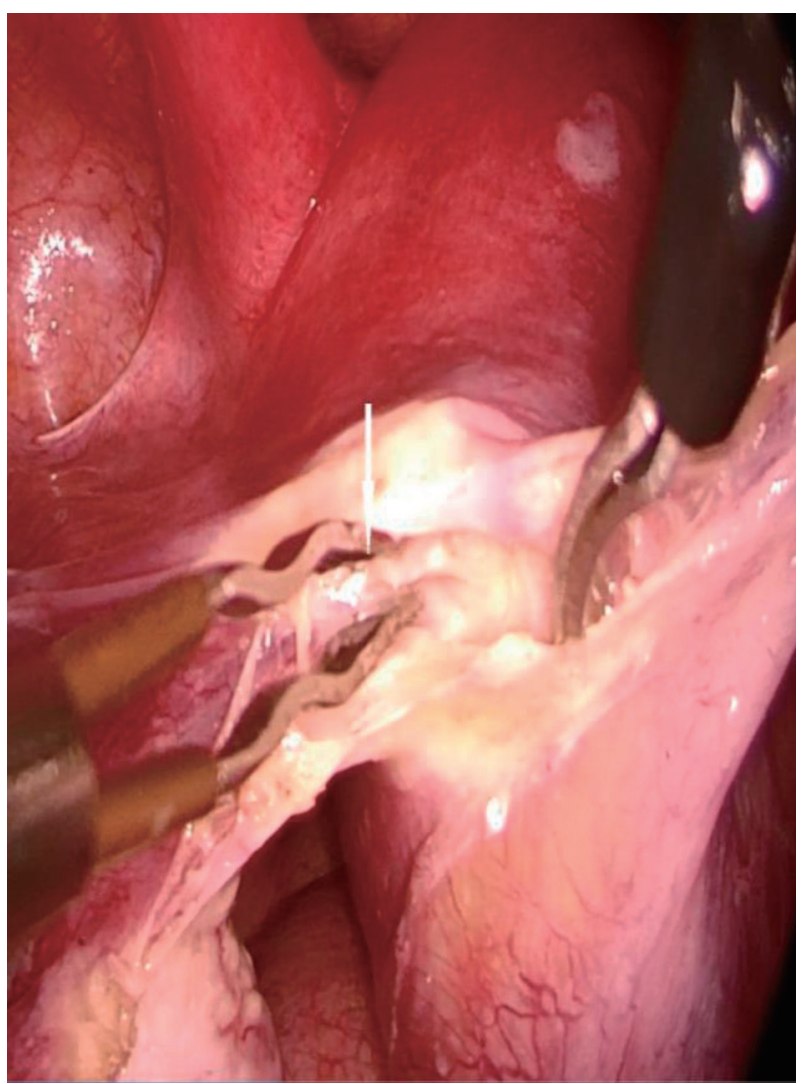

Figure 3. Bifurcation of uterine artery inferior to fallopian tube (white arrow).

that may suggest interstitial pregnancy include an empty uterus, gestational sac seen separately located less than $1 \mathrm{~cm}$ from the most lateral edge of uterine cavity and a thin myometrial layer surrounding the sac [8].

The common clinical signs and symptoms of presentation are similar to other types of ectopic pregnancy, commonly amenorrhea, abdominal pain and per vaginal bleeding. Those who present late with rupture may present with hemodynamic instability, due to excessive bleeding [9]. Traditionally, cornual resection or cornuostomy was the most common procedure performed for treatment of interstitial pregnancy but this modality was associated with more blood loss and loss of myometrium at this cornual area due to thermal injury and resection of myoma.

With advancement in imaging studies, surgical skills and techniques including laparoscopic surgical skills and experiences from the past, diagnoses are now typically made at an earlier gestational age and prior to rupture. This provides opportunities for more conservative medical and surgical treatments. A case series reported success in treating 52 patients with interstitial pregnancies laparoscopically, of whom, onethird conceived subsequently with no reported cases of uterine rupture or dehiscence. Laparoscopic wedge resection, laparoscopic cornuostomy and laparoscopic salpingectomy were carried out, with $17 \%$ requiring methotrexate treatment subsequently [10]. Considering the rarity of interstitial pregnancy, there were no randomized controlled trials or long-term follow- up comparing conservative management versus salpingectomy or cornuostomy versus cornual resection. There were also no studies found comparing laparoscopic conservative and radical treatment of tubal pregnancy. However, there are trends towards more conservative management recently. With the availability of trained skilled surgeon, laparoscopic approach can be considered due to the benefits of shorter recovery time, shorter hospitalization and lower health cost. Even if a woman presents with rupture and significant hemoperitoneum, laparoscopic surgery can still be safely conducted by experienced laparoscopic surgeon if the patient is hemodynamically stable. The main concern about treatment for interstitial pregnancy is the risk of uterine rupture in future pregnancy. It is because of cornuostomy and corneal resection-related myometrial loss. Uterine rupture has been reported with interstitial pregnancy, ranging from those who underwent treatment such as systemic methotrexate, cornual resection and salpingectomy, to those who had spontaneous resolution of the interstitial pregnancy. A literature review showed no difference for later recurrences with regards to the surgical approach. In fact, optimal suturing and a very limited use of electrocautery might play a more important role regarding later uterine rupture risks in future pregnancies.

Due to the rarity of the interstitial pregnancy in association with uterine fibroids, to our best knowledge, less than five cases reported interstitial pregnancy associated with uterine fibroids. The uterine fibroids range from small to $8 \mathrm{~cm}$, and treatments received include methotrexate therapy, evacuation of uterus, laparotomy cornuostomy, laparotomy cornual wedge resection and laparotomy myomectomy and cornuostomy. Recurrences were reported irrespective of mode of treatment. The numbers of cases reported were too small to conclude the treatment approach and risks of recurrence for counseling. However, it is noted that there is a higher risk in presence of uterine fibroids, especially myoma involving cornua region.

It is also known that myomectomy regardless of approach even though with suturing of the uterine incision may put patient at higher risk of uterine rupture. The postulated mechanism is due to underlying previously distended, defective uterine wall and thermal injury. Some suggest suturing the uterine wall defect after surgical management, for reinforcement. Others suggest an elective cesarean section should be taken for the future pregnancies following an interstitial pregnancy [2]. Nevertheless, there are reports of full-term delivery, both cesarean and vaginal delivery. To our knowledge, no long-term study follow-up on outcomes of those who had interstitial pregnancy to suggest optimum mode of delivery with respect to the treatment modality was undertaken. Optimal management depends on patient's fertility wishes.

We propose salpingectomy including interstitial portion of the tube as surgical option for non-ruptured interstitial pregnancy to minimize myometrial loss and thermal injury associated with conventional cornuostomy or cornual resection. This surgical technique can be done even with interstitial pregnancy with overlying myoma without myomectomy, as in our patient. Myomectomy and cornuoal resection involves incision of myometrial musculature resulting in weakening of the cornual region and thermal injury, subsequently increasing the risk of uterine rupture in future. 


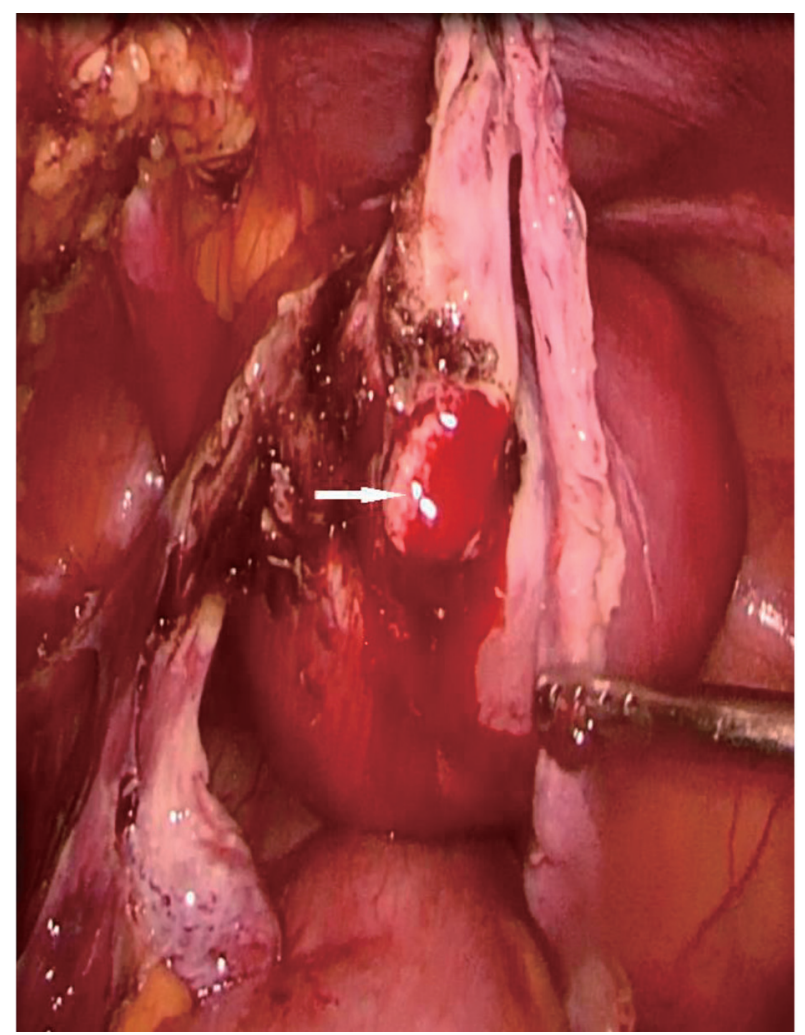

Figure 4. Exposure of product of conception at interstitial area.

In our case, we performed a salpingectomy by opening the broad ligament inferior to the fallopian tube and resecting the interstitial part of the tube without resecting the myometrium. At this junction, we had to ablate the rich vascular supply which was described as above. As we reached the interstitial fallopian tube, we dissected the anterior and posterior leaf of the broad ligament at the level of origin of the fallopian tube. At this point, the serosal layer of the myometrium was attached at the superior aspect of the tube but not at the inferior part of the tube. As we entered the avascular plane of fallopian tube at this juncture, the vasculature was identified and dissected away (Fig. 3). Fallopian tube was dissected completely and excised including interstitial fallopian tube. Product of conception was extruded upon exposure of interstitial tube (Figs. 4 and 5). Incision site was sutured with V-loc. We propose this surgical technique to avoid myometrial injury associated with cornuostomy, cornual resection and myomectomy, thus reducing morbidity associated with treatment of interstitial pregnancy. This method also reduces the intra-operative blood loss and subsequent long-term implications on pregnancy. This method may benefit women diagnosed with interstitial pregnancy, and those who have an overlying uterine fibroid and an interstitial pregnancy who require fertility in future.

\section{Conclusion}

In general, the available data are non-conclusive and conflicting in guiding appropriate counseling of patient with regards to

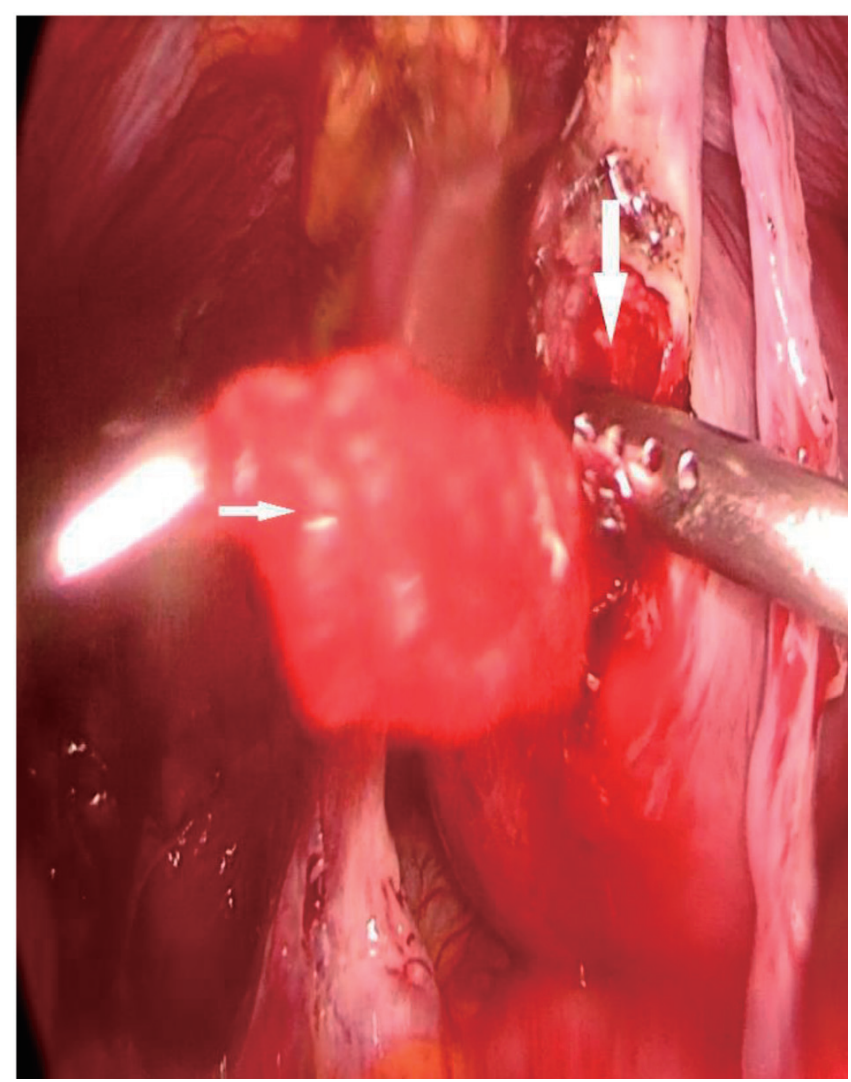

Figure 5. Product of conception (white arrow) extruded upon exposure of interstitial tube (thick white arrow).

treatment modality, impact on future pregnancy, mode of delivery in future pregnancy and future recurrences. Laparoscopic salpingectomy with complete removal of the interstitial part may be a new frontier in management of interstitial pregnancy. This method of surgery reduces morbidity associated with the conventional wedge resection or cornuostomy, such as blood loss, and potential risks of uterine rupture in future pregnancy. To the best of our knowledge, this is the first description of interstitial pregnancy with overlying uterine fibroid and successful laparoscopic salpingectomy, without cornuostomy or myomectomy.

\section{Acknowledgments}

None to declare.

\section{Financial Disclosure}

None to declare.

\section{Conflict of Interest}

None to declare. 


\section{Informed Consent}

Informed consent was obtained.

\section{Author Contributions}

Dr Ping Ling Ho: data and case collection, design, write-up, and finalization of write-up. Dr Janice Tung, Dr Siraj Shahul Hameed Mohamed and Prof. Dr Bernard Su Min Chern: supervision and final editing.

\section{References}

1. Farrer-Brown G, Beilby JO, Tarbit MH. The blood supply of the uterus. 1. Arterial vasculature. J Obstet Gynaecol Br Commonw. 1970;77(8):673-681.

2. Simpson JW, Alford CD, Miller AC. Interstitial pregnancy following homolateral salpingectomy. A report of 6 new cases and review of the literature. Am J Obstet Gynecol. 1961;82:1173-1179.

3. Radwan F, et al. Management of cornual (interstitial) preg- nancy. The Obstetrician \& Gynaecologist. 2007;9:249-255.

4. Wittich AC. Recurrent cornual ectopic pregnancy in a patient with leiomyomata uteri. J Am Osteopath Assoc. 1998;98(6):332-333.

5. Tulandi T, Al-Jaroudi D. Interstitial pregnancy: results generated from the Society of Reproductive Surgeons Registry. Obstet Gynecol. 2004;103(1):47-50.

6. Lee GS, Hur SY, Kown I, Shin JC, Kim SP, Kim SJ. Diagnosis of early intramural ectopic pregnancy. J Clin Ultrasound. 2005;33(4):190-192.

7. Chou MM, Tseng JJ, Yi YC, Chen WC, Ho ES. Diagnosis of an interstitial pregnancy with 4-dimensional volume contrast imaging. Am J Obstet Gynecol. 2005;193(4):15511553.

8. Soriano D, Vicus D, Mashiach R, Schiff E, Seidman D, Goldenberg M. Laparoscopic treatment of cornual pregnancy: a series of 20 consecutive cases. Fertil Steril. 2008;90(3):839-843.

9. Chan LY, Fok WY, Yuen PM. Pitfalls in diagnosis of interstitial pregnancy. Acta Obstet Gynecol Scand. 2003;82(9):867-870.

10. Ng S, Hamontri S, Chua I, Chern B, Siow A. Laparoscopic management of 53 cases of cornual ectopic pregnancy. Fertil Steril. 2009;92(2):448-452. 